\title{
DECLARACIÓN POR EL DESARROLLO DE LA PSICOLOGÍA CLÍNICA EN ESPAÑA
}

La Psicología Clínica se encuentra en un momento histórico en el que actores, concepciones, e intereses divergentes buscan influir en la orientación de su evolución futura. En atención a la importancia de la función que asumen los psicólogos/as clínicos por desempeñar su profesión en un área de especial valor social como es la intervención especializada en el ámbito de la salud y enfermedad humanas, las Asociaciones Científicas y Profesionales firmantes pensamos que ha llegado el momento de promover esta declaración, en la que el recuerdo de nuestra historia sirva de punto de partida para poner en valor los logros alcanzados y resaltar lo mucho que queda por lograr y consolidar. Con ella pretendemos impedir que se produzca un debilitamiento de los procedimientos y medios existentes en nuestro país para la obtención y certificación de la competencia de los psicólogos/as clínicos, y llamar la atención del colectivo profesional y científico, de las Instituciones y Administraciones Públicas y del conjunto de la sociedad sobre la necesidad de actuaciones favorecedoras del desarrollo de esta especialidad sanitaria.

Es por ello que consideramos y proponemos:

1. El desarrollo de la Psicología Clínica en España como especialidad sanitaria de la Psicología está históricamente unido al progreso social y modernización de la sociedad española. A ello contribuyeron sin duda muchos profesionales de la Psicología tanto del ámbito del ejercicio privado como del público, así como asociaciones científicas y profesionales, académicos/as, profesionales de otros campos del saber, y asociaciones ciudadanas defensoras de la sanidad pública. El interés en proporcionar una atención de calidad a las necesidades y derechos sanitarios de la población de forma acorde con el conocimiento científico, sustentó hasta la actualidad la legitimidad de las propuestas y acciones de los psicólogos/as.
En su proceso de construcción como ámbito de saber y de ejercicio profesional autónomo, la Psicología Española generó unas señas de identidad que hoy han de ser resaltadas: multidisciplinariedad, autoexigencia, compromiso ético, respeto a la diversidad teórico-técnica, cientificismo, voluntad de servicio público y compromiso con el desarrollo del Sistema Nacional de Salud (SNS).

2. El reconocimiento legal de la especialidad sanitaria de Psicología Clínica, y con ello su institucionalización, constituyó un avance histórico de la Psicología en España, cuyo resultado fue saludado unánimemente por sus representantes académicos y profesionales.

Los motivos y actitudes que impulsaron su nacimiento y sustentaron en la Psicología Española la unitaria labor colectiva que lo facilitó, poseen plena validez y necesidad cuando, como en el momento actual, la tarea es de consolidación y de desarrollo de un progreso por el que todos aún recientemente nos congratulamos.

3. El momento actual de la Psicología Clínica expresa una compleja realidad en la que bajo progresos evidentes existen carencias y riesgos. No puede ser de otro modo.

En este contexto, lo que fue un proceso prolongado en el tiempo y lleno de enseñanzas, trabajosamente elaborado, culminado inteligentemente en la creación oficial de la Especialidad después de sortear múltiples dificultades, aparece hoy ensombrecido por una sostenida confusión que ha generado preocupación en la comunidad científico-profesional de la Psicología. Las explícitas interpretaciones ministeriales de la Ley 44/2003 (Ley de Ordenación de las Profesiones Sanitarias) y las propuestas de modificación del R.D. 1277/2003 (por el que se 
establecen las bases generales para autorización de centros, servicios y establecimientos sanitarios) realizadas por la Comisión Nacional de la Especialidad de Psicología Clínica (CNEPC) y las asociaciones que suscriben esta declaración, cierran en lo sustancial el capítulo de acciones aclaratorias sobre el estado de la normativa legal y su valoración. Hay que abrir nuevas líneas de reflexión y acción que permitan el progreso.

Es fundamental para ello esforzarse en situar en perspectiva nuestra evolución y nuestros problemas como especialidad y disciplina, evitando la respuesta precipitada y urgente a cuestiones que tienen raíces y protagonistas antiguos y que, como la definición de la formación sanitaria especializada o el ámbito del ejercicio profesional, tienen gran repercusión colectiva a largo plazo.

4. La institucionalización de la Psicología Clínica en España fue realizada de acuerdo con los procedimientos y la forma que tiene nuestro sistema legal, educativo y sanitario de ordenar las especialidades sanitarias y de regular su titulación y ejercicio profesional. Ello conllevó la unión inseparable de la acreditación de la capacidad especializada del Psicólogo Clínico (mediante título oficial reconocedor de derechos y obligaciones) con la culminación de un proceso formativó tan eficaz como prestigioso (Sistema de Residencia), a realizar en el marco del Sistema Nacional de Salud.

Esta formación se realiza a través de la práctica asistencial tutelada y de responsabilidad creciente, desempeñada en un circuito de rotación formado por una red de servicios sanitarios diversificados y multiprofesionales que tienen responsabilidad sanitaria sobre población de un ámbito territorial definido, y que al cumplir requisitos específicos y necesarios para ser Unidades Docentes Acreditadas (UDA) para la formación especializada se constituyen en instrumento fundamental para la obtención de competencia clínica. Este modelo ha demostrado su bondad a lo largo de estos años, hasta el punto de haber sido base y referente para programas de formación de otras especialidades sanitarias.

Entendemos que este proceso garantiza la formación clínica adecuada y coherente con nuestra tradición de autoexigencia y sustenta nuestra legítima aspiración a desarrollarnos profesionalmente en igualdad de condiciones que otros facultativos sanitarios.

5. Es necesario potenciar el desarrollo del sistema PIR para la formación de especialistas sanitarios en Psicología Clínica. $\mathrm{Su}$ existencia es un progreso pero presenta en la actualidad insuficiencias que es responsabilidad de las Administraciones Sanitaria y Educativa resolver. Urge la aprobación del Nuevo Programa de Formación de la Especialidad con su ampliación a cuatro años, el desarrollo de las áreas de capacitación específica, la mejora de los instrumentos para garantizar la calidad y evaluación docentes, así como el incremento de las plazas PIR acreditadas y ofertadas en la convocatoria anual para el acceso al Programa Formativo.

Así mismo, debe incorporarse ya de forma normalizada la CNEPC a los diferentes órganos ministeriales que tienen funciones relacionadas con el desarrollo formativo y profesional de los recursos humanos del SNS.

6. El ejercicio profesional de la Psicología Clínica en España se caracteriza por su diversidad y complejidad crecientes. La competencia del colectivo profesional que lo sustenta es elevada. La participación e interés de los profesionales en su actualización formativa es muy satisfactoria. A pesar de su crecimiento y capacidad asistimos en los últimos tiempos a desarrollos legislativos incoherentes con normas anteriores, desconsiderados con los conocimientos profesionales y las necesidades sanitarias y lesivos para la Psicología Clínica entre los que destaca especialmente el R.D. de autorización de centros sanitarios. Los citados desarrollos han de ser adecuadamente cuestio- 
nados y modificados para, además de otros objetivos, garantizar el reconocimiento de las funciones profesionales ya consolidadas en la práctica profesional.

7. Los psicólogos clínicos accedieron en su mayoría al SNS durante el proceso de Reforma de los dispositivos de atención a la Salud Mental (Reforma Psiquiátrica) desarrollado en nuestro país hasta bien avanzada la década de los 90 . Desde hace años asistimos a la práctica congelación de la dotación de estos profesionales en la red sanitaria pública. La cantidad relativamente reducida de los mismos en el sistema y el volumen y diversidad de la demanda a la que han de responder, constituye tanto una injusta limitación de la prestación de sus servicios a los usuarios de la Sanidad Pública, como un grave inconveniente para el desarrollo científico y técnico de la Psicología Clínica y el incremento de su capacidad de respuesta sanitaria relevante.

Es urgente en el SNS satisfacer la necesidad de aumento de estos profesionales así como promover su incorporación a otros ámbitos sanitarios además del de la atención a la Salud Mental.

Por otra parte, la inadecuada y obsoleta definición de su categoría administrativa exige inmediata modificación acorde con su condición de facultativo especialista sanitario, tal y como establece la disposición adicional segunda del Real Decreto $2490 / 98$ por el que se crea y regula la Especialidad de Psicología Clínica.

8. La Universidad, en el contexto actual de cambio de los estudios universitarios de Psicología enmarcado en el proceso de Convergencia Europea, ha de velar por la compatibilización de las necesarias modificaciones en la estructura, contenidos y métodos de los estudios universitarios, con la existencia de un procedimiento en España (Sistema PIR) de elevada exigencia para la obtención de la capacidad y el título de Especialista en Psicología Clínica. Este sistema no sólo constituye una garantía de calidad for- mativa sino también de igualdad en las posibilidades de acceso al Sistema Formativo, al reducir las posibilidades de que exista entre los licenciados otra discriminación que no sea la que se deriva de sus méritos y capacidades.

Consecuentemente con ello, valoramos que la Universidad ha de aumentar su participación e implicación en el desarrollo de la especialidad de Psicología Clínica adoptando en la actualidad las siguientes medidas: ampliación de la formación de los futuros licenciados en las materias relacionadas con el currículo formativo de la Especialidad, vinculación formal de los Psicólogos Especialistas en Psicología Clínica de la red asistencial con el desarrollo de las prácticas pre-profesionales (practicum), reivindicación y creación de las plazas vinculadas con el SNS para profesores universitarios en posesión del título de especialista, potenciación de la formación postgrado y cooperación en tareas de investigación con los psicólogos clínicos de la red asistencial.

9. El impulso necesario para dar continuidad al desarrollo innegablemente positivo de la Psicología Clínica en España de tal forma que la misma continúe en su avance hacia mayores niveles de capacidad de respuesta a las necesidades de salud de la población, de presencia y decisión sanitarias, de cualificación y competencia profesional y de autoexigencia y compromiso social, reclama de sus organizaciones científicas y profesionales altura en las ambiciones, coherencia y rigor en sus análisis, y responsabilidad en sus propuestas y acciones. Corresponde también a cada psicólogo clínico la realización de su propia contribución al crecimiento de la disciplina científica y profesional de la que es parte, mediante su práctica rigurosa, ética, comprometida y respetuosa con quienes necesitan de sus servicios docentes y/o asistenciales.

No ha de aceptarse la devaluación de la Especialidad. No ha de permitirse que se creen condiciones que puedan favorecer el cuestionamiento de las capacida- 
des que poseen quienes la ostentan, ya sea por reducción de las exigencias de demostración del mérito o del rigor y claridad de los procedimientos establecidos para su obtención, ya sea por insuficiente dotación de recursos para la formación de los psicólogos residentes o para la adecuada práctica de los profesionales.

10. Nunca fue posible el progreso sin la existencia de tensiones y contradicciones de intereses.

Tiene que darse continuidad al desarrollo de la Psicología Clínica en España desde la conciencia y la estima de nuestra historia y nuestra identidad. Se ha de procurar, desde los avances logrados, ampliar la unidad en el esfuerzo sobre la transparencia de los motivos y el diálogo de las diferencias.

Pero hemos de recordar que el fundamento de la legitimidad y de la razón de nuestras propuestas y reivindicaciones se encuentra tanto en la utilización de procedimientos democráticos para su definición y defensa, como en el valor que nuestra actuación y competencia profesional poseen para mejorar la vida de aquellos que, con su enfermedad y malestar, reclaman nuestra ayuda.

Con el fin de avanzar en la materialización de estas propuestas a través de las acciones futuras que sean necesarias, acordamos la constitución de la Mesa por el Desarrollo de la Psicología Clínica, inicialmente conformada por las organizaciones firmantes y abierta a incorporaciones futuras de aquellas otras que compartan esta Declaración.

Madrid, 17 de septiembre de 2004

Asociación Española
de Neuropsiquiatría (AEN)
Asociación Española de Psicología Clínica
y Psicopatología (AEPCP)
Asociación Nacional Psicólogos Internos
Residentes (ANPIR)
Colegio Oficial de Psicólogos de Andalucía
Occidental (COPAO)
Colexio Oficial de Psicologos
de Galicia (COPG)
de Galicia (COPG) 